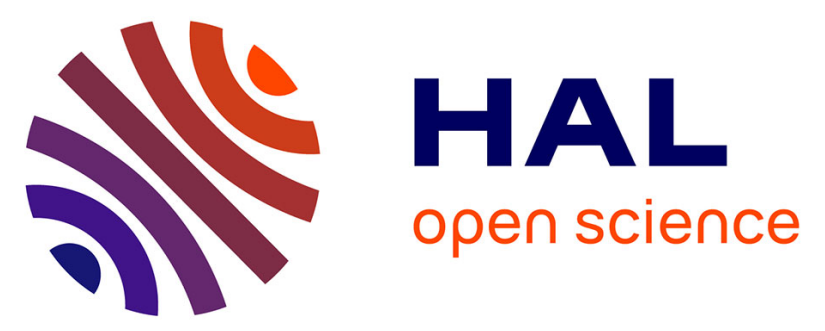

\title{
Toward "Pseudo-Haptic Avatars": Modifying the Visual Animation of Self-Avatar Can Simulate the Perception of Weight Lifting
}

\author{
David Antonio Gómez Jáuregui, Ferran Argelaguet Sanz, Anne-Hélène \\ Olivier, Maud Marchal, Franck Multon, Anatole Lécuyer
}

\section{To cite this version:}

David Antonio Gómez Jáuregui, Ferran Argelaguet Sanz, Anne-Hélène Olivier, Maud Marchal, Franck Multon, et al.. Toward "Pseudo-Haptic Avatars": Modifying the Visual Animation of Self-Avatar Can Simulate the Perception of Weight Lifting. IEEE Transactions on Visualization and Computer Graphics, 2014, 20 (4), pp.654-661. 10.1109/TVCG.2014.45 . hal-00990280

\section{HAL Id: hal-00990280 https://hal.inria.fr/hal-00990280}

Submitted on 14 May 2014

HAL is a multi-disciplinary open access archive for the deposit and dissemination of scientific research documents, whether they are published or not. The documents may come from teaching and research institutions in France or abroad, or from public or private research centers.
L'archive ouverte pluridisciplinaire HAL, est destinée au dépôt et à la diffusion de documents scientifiques de niveau recherche, publiés ou non, émanant des établissements d'enseignement et de recherche français ou étrangers, des laboratoires publics ou privés. 


\title{
Toward "Pseudo-Haptic Avatars": Modifying the Visual Animation of Self-Avatar Can Simulate the Perception of Weight Lifting
}

\author{
David Antonio Gómez Jáuregui, Ferran Argelaguet, Anne-Hélène Olivier, \\ Maud Marchal, Franck Multon and Anatole Lécuyer,
}

\begin{abstract}
In this paper we study how the visual animation of a self-avatar can be artificially modified in real-time in order to generate different haptic perceptions. In our experimental setup, participants could watch their self-avatar in a virtual environment in mirror mode while performing a weight lifting task. Users could map their gestures on the self-animated avatar in real-time using a Kinect. We introduce three kinds of modification of the visual animation of the self-avatar according to the effort delivered by the virtual avatar: 1) changes on the spatial mapping between the user's gestures and the avatar, 2) different motion profiles of the animation, and 3) changes in the posture of the avatar (upper-body inclination). The experimental task consisted of a weight lifting task in which participants had to order four virtual dumbbells according to their virtual weight. The user had to lift each virtual dumbbells by means of a tangible stick, the animation of the avatar was modulated according to the virtual weight of the dumbbell. The results showed that the altering the spatial mapping delivered the best performance. Nevertheless, participants globally appreciated all the different visual effects. Our results pave the way to the exploitation of such novel techniques in various VR applications such as sport training, exercise games, or industrial training scenarios in single or collaborative mode.
\end{abstract}

Index Terms-Self-animated avatar, avatar-based physical interaction, pseudo-haptic feedback, perception of motion dynamics

\section{INTRODUCTION}

Avatar-based interaction has become increasingly popular in recent years in virtual reality applications [12]. The avatar is the embodied manifestation of the user in the virtual environment. Commonly, the users' actions (gestures or full-body motions) can be directly mapped to the avatar, enabling a direct control. In addition to enable interaction, users can identify themselves with a self-animated avatar which provides a sense of identity and existence of the user inside the virtual world [3].

In this work, we explore how the mapping between the user's gestures and the self-avatar animations can be altered in order to physicalize the interaction through a pseudo-haptic feedback approach [15]. Pseudo-haptic feedback combines visual feedback in a synchronized way with the user's actions during an interaction process in order to create a visuo-haptic illusion. While pseudo-haptic has been the focus of many experiments that have simulated various haptic properties such as stiffness or friction, it has been mainly targeting desktop based-interaction. Although few works has explored virtual reality or mixed reality setups $[24,22]$ there is no existing work considering avatar-based interaction. The usage of pseudo-haptic effects in avatarbased interaction is challenging as the user interacts with the system using unconstrained gestures in free space. In current avatar-based systems the user's gestures are mapped directly to the avatar. Thereby, avatar animations are perceived as effortless; no matter the task or the nature of the interacted objects. As a first study we are exploring how a weight lifting task can be enhanced through pseudo-haptic feedback in order to enable the user the perception of different weights and thus enable the user to discriminate objects according to their physical

- David Antonio Gómez Jáuregui is with Hybrid Research Team at Inria Rennes. E-mail: david_gomez1380@yahoo.com.mx.

- Ferran Arguelaguet is with Hybrid Research Team at Inria Rennes. E-mail: fernando.argelaguet_sanz@inria.fr.

- Anne-Hélène Olivier is with Mimetic Research Team at Inria Rennes. E-mail: anne-helene.olivier@inria.fr.

- Maud Marchal is with Hybrid Research Team at Inria Rennes. E-mail: maud.marchal@irisa.fr.

- Franck Multon is with Mimetic Research Team at Inria Rennes. E-mail. franck.multon@irisa.fr.

- Anatole Lécuyer is with Hybrid Research Team at Inria Rennes. E-mail: Anatole.Lecuyer@inria.fr. properties. We propose three different approaches in order to adapt the animation of the avatar (visual feedback) according to the users' gestures and the effort of the underlying task. The approaches are based on altering three different aspects of the animation:

- The spatial mapping between the users' gestures and the avatar. Amplifying/reducing the user motion by changing the control/display ratio. For example, in order to lift a heavier object, the user would be required to perform a wider gesture than when lifting a lighter object.

- The motion profile of the animation. When lifting objects with different weights the speed and acceleration of the avatar animation would vary. Recorded motion capture data was used in order to provide a realistic animation.

- The posture of the avatar (upper-body inclination). The angle of inclination of the avatar during the lifting gesture would be dependent on the weight of the virtual object.

In order to validate our approach, we conducted an experiment in which participants were presented with a weight discrimination task. Users were asked to order four virtual dumbbells according to their weight. Participants had to lift each virtual dumbbell by controlling a self-animated avatar in real-time by physically performing a weight lifting gesture. The animation of the avatar while lifting the virtual dumbbell was modulated according to the proposed techniques. The results of the evaluation showed that the technique modulating the user's motion enabled the user to better discriminate the weight of the virtual dumbbells. The potential applications of our approach range from full-body motion-based videogames, training simulators and collaborative virtual environments.

The remainder of the paper is organized as follows. Section 2 reviews related work on influence of visual information, pseudo-haptic feedback and physical interaction through avatars. Section 3 details our proposed approach for pseudo-haptic avatars. Section 4 describes and discusses our user evaluation study. Finally, conclusions and future work are described in Section 5.

\section{Related WORK}

\subsection{Avatar-based Interaction}

Avatar-based interaction has received great interest since to interact in virtual environments [12]. An avatar describes the manifestation of 
the user in the virtual world. In this way, a self-avatar enables direct interaction, facilitates the mobility of the user in the virtual world and provides a strong sense of identity [9]. In addition, avatar-based interaction has also been found to improve interaction performance [20] and distance estimation [1]; people tend to underestimate distances in virtual environments.

Collaborative interaction can also benefit from avatar-based interaction. The embodiment of the actors in the collaboration in virtual avatars enables the exchange of information at different levels, such as gestural communication [4] and knowledge transfer [21]. Dodds et al. [4] investigated the importance of self-animated avatars in a communication game between two users. In contrast to static avatars, the ability of control the animation of the avatars in real-time showed a significant improvement in the quality of the communication between users. This effect occurred mainly in the third-person perspective where users were able to view their own avatars. In the scope of training systems, Ogawa et al. [21] proposed a real-time physical instructional support system for martial arts. The instructors and the learners, who are in remote places, interact with the system through body gestures. To asses learners, the instructor can superimpose his avatar with a learner's avatar to better demonstrate the motions to the learners.

In this paper, though, we are interested in how avatar interaction is perceived by the user. Recent studies suggest that perception of human interaction is similar in real and virtual environments. For example, Hoyet et al. [11] compared the perception of participants between real weight lifting motions (videos) and corresponding captured motions applied to an avatar. Results showed that subjects were able to distinguish masses of dumbbells lifted by real (videos) and virtual humans with similar accuracy. However, despite the large popularity of avatar-based interaction (e.g. training simulators, videogames, virtual reality), there are few research works that take advantage of the sense of identity of users with avatars in order to enhance physical interaction with virtual objects.

Body ownership and out-of-body experiences have been largely explored in the context of immersive virtual reality. Existing studies have explored up to which extend a virtual avatar $[18,27]$ (or a virtual limb [26]) can be recognized by the user as his own body and its correlation with the level of presence within the virtual environment. As a first study, Slater et al. [26] reproduced the "rubber hand illusion" in a virtual reality platform and demonstrated that a feeling of ownership of a virtual arm can be achieved if the appropriate multi sensory correlations are provided. Other studies has been performed focusing on how the physical appearance of the avatar can influence the strength of the body ownership illusion [13] or the influence of the avatar's hands on object size perception [19]. This studies observed that coherency between the virtual avatar, the user and the task performed plays an important role on the strength of the body ownership illusion and in user performance [13]. Furthermore, the size of the avatar with respect to the virtual objects can also influence users' perception of the virtual environment [19].

\subsection{Influence of visual information}

The influence of visual information on the perception of object properties has been widely studied. More generally, several authors have investigated the influence of vision on touch senses. Various studies have found that vision frequently dominates touch when judging size [10, 14] or shape [25]. Srinivasan et al. [28] demonstrated that visual information of displacement has a compelling impact on the perceived stiffness of virtual springs. In a posterior study, Heller et al. [10] found that dominance relations vary with the speed and precision of the response measure and modality and that there are circumstances that promote reliance on touch or on vision. Based on previous studies, Ernst and Banks [7] proposed a model to estimate the degree to which vision or haptic dominates in visual-haptic tasks.

The influence of visual information has been applied to alter the users perception of virtual and real objects in Mixed Reality (MR) systems. Omosako et al. [23] investigated the influence of visual information on Center-of-Gravity (CoG) perception. In this study, the authors conducted experiments to examine the influence of superim- posing virtual objects having different CoG positions onto real objects with different mass. The results confirmed that CoG perception is biased by the shape of virtual objects. Ban et al. [2] proposed a system to alleviate fatigue during handling medium-weight objects and augmenting our endurance by affecting the weight perception using augmented reality technology. The weight perception was modified by changing the apparent brightness of objects. The results indicated that the system reduced fatigue during the handling task by eliminating the need to use excess force for handling objects.

\subsection{Pseudo-haptic feedback}

Pseudo-haptic feedback is a technique that leverages the visual dominance on the perception of object properties in visual-haptic interactions [15]. The objective of pseudo-haptic feedback is to simulate haptic sensations, without necessarily using a haptic interface, such as stiffness [16] or image texture [8].

Traditional usages of pseudo-haptic feedback has focused on modulating the mapping between the actions of the user and the feedback provided by the system by altering the Control/Display ratio. Lécuyer et al. [17] simulated friction with a virtual cube moved horizontally across a simple virtual environment using only a 2D mouse and a Spaceball. The cube was decelerated or accelerated by altering the Control/Display ratio. In a posterior study, Dominjon et al. [5] evaluated the influence of the Control/Display ratio on the perception of mass of manipulated objects in a virtual environment. In their experiments, participants were asked to identify the heaviest object between two virtual balls through a haptic interface while looking to its virtual representation. The motion of the virtual balls was altered artificially according to their virtual weight. The results showed that the Control/Display ratio significantly influenced the results of mass discrimination and sometimes even reversed them.

In traditional pseudo-haptic approaches the user interacts with the system through a virtual prop (e.g. mouse cursor, sphere). In contrast, the work of Pusch et al. [24] explored whether pseudo-haptic feedback can be achieved if the interaction tool is the user's hand on itself. Through a see-through HMD system they manipulated the motion of the user's hand in order to simulate a force field effect. This results showed that a pseudo-haptic effect can also be achieved even if the user is interacting in free space with gestures.

In contrast to existing works, in this paper we are focusing whether the pseudo-haptic effect can be delivered when the visual information come from a self-animated avatar representing the user embodiment in the virtual environment.

\section{The Pseudo-Haptic Avatar approach for Weight LIFTING SIMULATION}

Our pseudo-haptic avatar approach is based on altering the mapping between the users' gestures and the gestures of the virtual avatar in order to generate a haptic perception. In this work we focus on physicalizing avatar-based interaction by enabling the user to perceive the effort delivered by the avatar while performing a weight lifting task. Specifically, the task considered is the lift of a dumbbell with both arms (see Figure 1). In this section, we first detail the protocol followed in order to obtain a realistic avatar animation of the weight lifting task (motion capture data). Then, we describe the three different visual feedbacks proposed which were inspired by the data obtained during the motion capture session and existing pseudo-haptic approaches.

\subsection{Motion capture of weight lifting}

The animation of the avatar was computed using motion capture data. We chose this technique to ensure a realistic lifting gesture animation. One volunteer was recruited for this purpose. The volunteer was $1.78 \mathrm{~m}$ tall and 30 years old. He had no known pathology which would affect his motion. The volunteer gave written and informed consent before his inclusion and the study conformed to the declaration of Helsinki. During the recording, the volunteer stood still in front of a table. The task was to lift with both hands in pronation a dumbbell 

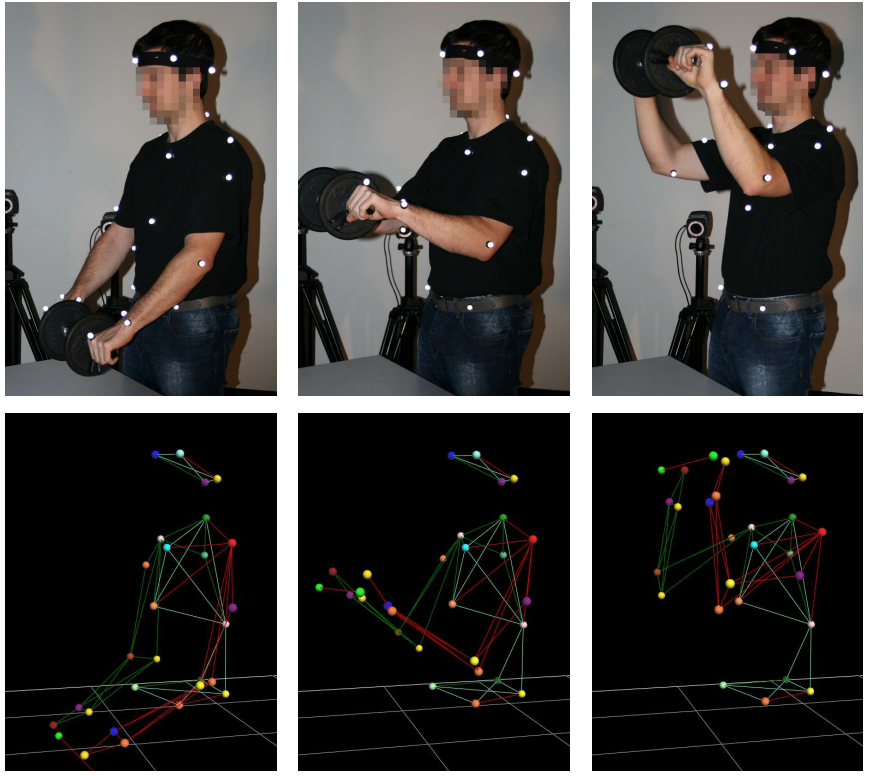

Fig. 1. Motion capture data process. Top, data recording of the real weight lifting gesture task. Bottom, 3D reconstruction of the weight lifting gesture by Vicon IQ software.

Table 1. Relationship between the anatomical landmarks tracked during the motion capture session and the articilations joints of the virtual avatar. Avatar's articulation joints are showed in Figure 2.

\begin{tabular}{|c|c|}
\hline Anatomical landmarks & Articulation joints \\
\hline \hline Xiphoid process & Spine (1) \\
\hline Suprasternal notch & Shoulder center (2) \\
\hline Frontal and occipital bones & Head (3) \\
\hline Left acromion & Left Shoulder (4) \\
\hline Right acromion & Right Shoulder (5) \\
\hline $\begin{array}{c}\text { Left medial epicondyle of the } \\
\text { humerus and left head of radial bone }\end{array}$ & Left Elbow (6) \\
\hline $\begin{array}{c}\text { Right medial epicondyle of the } \\
\text { humerus and right head of radial bone }\end{array}$ & Right Elbow (7) \\
\hline Left Radial and left ulnar styloid process & Left Wrist (8) \\
\hline Right Radial and right ulnar styloid process & Right Wrist (9) \\
\hline
\end{tabular}

placed on the table (see Figure 1 top). We manipulated the weight of the dumbbell $(2,6,10$, and $14 \mathrm{~kg})$. A total of 12 trials (4 weights * 3 repetitions) were performed by the volunteer. 27 reflective markers were attached to the volunteer's upper body on standardized anatomical landmarks [29]. 3D kinematic data were recorded using the optoelectronic motion capture device Vicon MX-40 (Oxford Metrics) with 12 high resolution cameras at a sampling rate of $120 \mathrm{~Hz}$.

After the motion was captured, the reconstruction of the 3D positions (world coordinates) as well as the labeling of the reflective markers were processed using Vicon IQ software (see Figure 1 bottom). Then, the 3D positions for each joint articulation of the avatar were obtained by computing the centroid of the respective group of markers [6]. Table 1 shows the corresponding group of anatomical landmarks used to obtain each joint position of the avatar. Given the joint positions, the 3D posture of the avatar is easily obtained by computing the joint angles (see Figure 2). The 3D postures obtained for each weight of the dumbbell are used to animate the virtual character. Thus, for each weight we have a corresponding avatar animation.

\subsection{Visual effects proposed for pseudo-haptic avatar}

In our approach, the avatar animation obtained from the motion capture data is synchronized with the gestures of the user and then altered

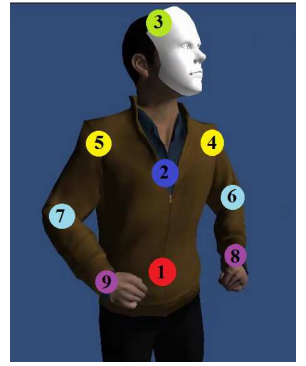

Fig. 2. Virtual avatar used and the position of the nine articulation joints used to animate it.

or distorted in three different ways. Users can perceive different sensations by observing the altered motion of their self-animated avatar while interacting with the virtual object. In order to provide an avatar animation according to the weight lifted, we have considered three animation properties: the motion profile, the posture of the avatar and the Control/Display ratio between the user's gestures and the avatar animation. Each visual effect will be explained with respect to the motion capture data of the lifting effort (section 3.1).

\subsubsection{First effect: Motion profile}

In this first visual effect, we explore whether users are able to perceive the weight of virtual objects only through the changes of the avatar animation from the original motion capture data. In this case, we have used the lifting motions obtained for different weights of real objects in order to simulate the respective weights in the virtual object.

The user gestures are synchronized in real-time with the avatar animation that corresponds to the weight of the object being manipulated; user gestures are captured in real-time using a Kinect. In order to synchronize the recorded animation with the user's gestures, considering that the vertical position of the wrist during the lifting operation is monotonic, the vertical range of the users' wrist is associated with the postures (frames) of the recorded animation. If we consider that the animation is split in a set of postures $P$ given the current position of the wrist $Y$ the posture of the avatar is defined by Equation 1:

$$
P=P_{0}+\left(P_{F}-P_{0}\right) \frac{Y-Y_{0}}{Y_{F}-Y_{0}}
$$

where $Y_{0}$ and $Y_{F}$ are the initial and maximum vertical wrists position (y-coordinate) of the user motion. $P_{0}$ and $P_{F}$ are the initial avatar posture and final avatar postures of the lifting motion for a specific weight. The vertical wrists positions are obtained by computing the centroid between the left and right wrists vertical positions (y coordinates). In the lifting motion, the initial and maximum vertical wrist positions are defined by the vertical positions of the waist and head of the user respectively. Both positions are obtained with a Kinect sensor during a calibration step.

Figure 3 shows the motion profile for the different weights considered. For each weight, the lifting effort is characterized by the position of the wrists in the recorded animation and its evolution over time. The different animation profiles are expected to enable users to perceive different weights.

\subsubsection{Second effect: Angle of inclination}

The second visual effect consists in modifying artificially the inclination of the avatar in order to simulate different lifting efforts. As observed in motion capture data, the avatar leaned more when the dumbbell was heavier. This visual feedback aims to show effort of the avatar via its posture. We straightforward associate the lifting effort to the angle of inclination of the avatar.

In order to simulate a lifting effort, two types of inclination were used: frontward and backward. Frontward inclination was used when the avatar is starting to lift the object while backward inclination was used when the avatar is raising the object. The inclination of the avatar 


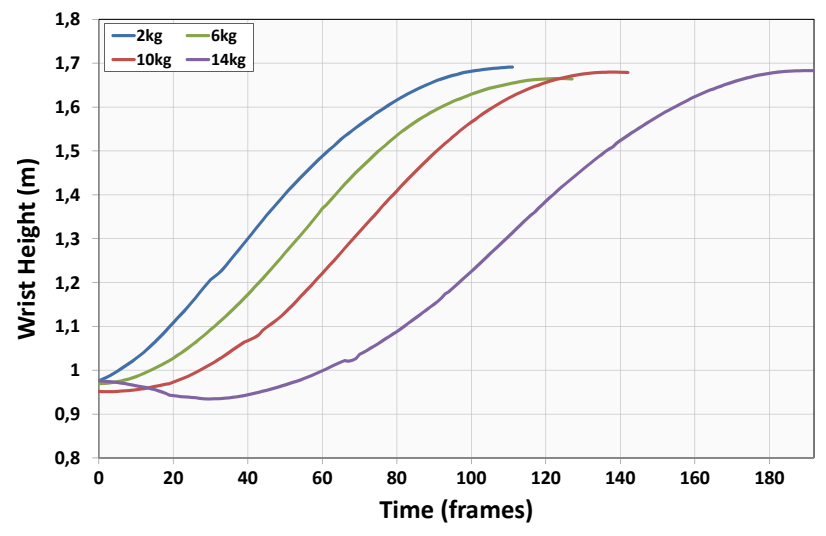

Fig. 3. Vertical trajectory of the wrists position over time for the four motion profiles considered (real motion capture data).

is simulated using a pitch rotation in the spine articulation joint (see Table 1 and Figure 2). The pitch rotation is computed using the following equation.

$$
\alpha=\arctan \frac{(Z+i)-Z_{S}}{Y-Y_{S}}
$$

where $\alpha$ is the angle of inclination of the avatar. $Y_{S}$ and $Z_{S}$ are the coordinates (in the $y$ - $z$ plane) of the spine articulation joint. $Y$ and $Z$ are the coordinates of the shoulder center articulation joint. An offset value $i$ is added to the $\mathrm{z}$-coordinate $Z$ in order to control the angle of inclination of the avatar. A frontward inclination is obtained with a positive offset value. In the contrary case, a negative offset value is used to have a backward inclination. Then, the lifting effort is simulated by interpolating the angle of inclination along the lifting motion obtained (see Section 3.1). In this case, the offset value $i$ is interpolated using the following equation.

$$
i=i_{0}+\left(i_{F}-i_{0}\right) \frac{P-P_{0}}{P_{F}-P_{0}}
$$

where $i_{0}$ and $i_{F}$ is the initial and final value of the offset that defines the full pitch rotation of the spine articulation joint. These offset values can be arbitrarily defined in order to show a consistent lifting effort for a given weight. For example, for a forward inclination the initial value $i_{0}$ can be defined as 0 and the final value $i_{F}$ must be greater than 0 (depending of the amount of rotation that we want to show for a given weight). For a backward inclination the initial value $i_{0}$ is the final value of the forward inclination and the final value $i_{F}$ must be less than 0 . $P_{0}$ and $P_{F}$ are the initial avatar posture and final avatar postures of the lifting motion for a specific weight. $P$ is the current posture obtained from the current wrist position of the user (see Equation 1) In this equation, a specific offset $i_{F}$ is chosen in order to simulate a different lifting effort. Thus, the larger the value of the offset $i_{F}$, the larger the angle of inclination and therefore, the heavier the weight simulated.

Considering a lifting motion of $N$ postures, a frontward inclination (positive offset value) is computed in the first $n$ postures while the backward inclination (negative offset value) is computed in the remaining $N-n$ postures. Figure 4 shows the evolution of the angle of inclination of the avatar for the lifting motion of each weight dumbbell. Figure 5 shows the sampled postures from the avatar animation resulted by interpolating the angle of inclination during the lifting motion (maximum value of the forward inclination).

\subsubsection{Third effect: Control/Display ratio of the avatar motion}

This third visual effect consists in increasing (or decreasing) the amplitude of the avatar motion. The principle is similar to classical pseudohaptic feedback approaches where the visual motion of a manipulated virtual object is amplified or reduced when compared to the motion of the user hand $[15,5]$. Thus, we have transposed this principle here to the visual lifting animation of self-avatars. In the proposed approach,

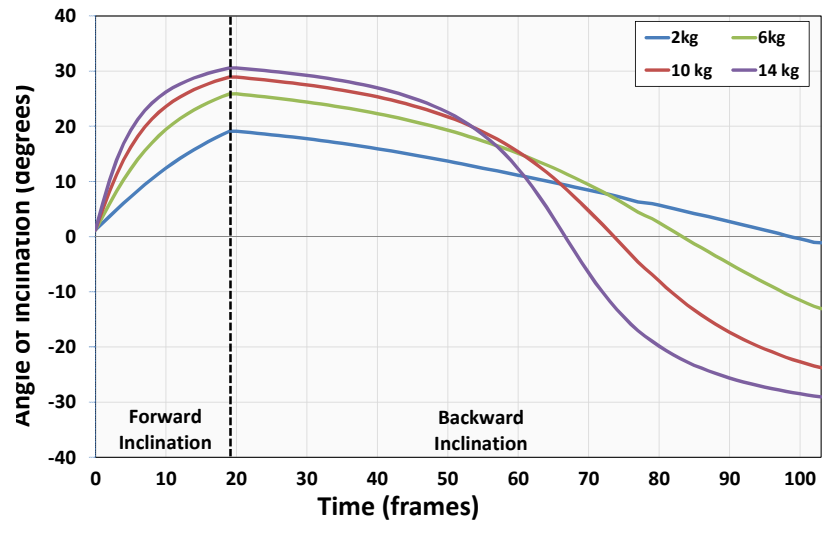

Fig. 4. Temporal evolution of the angle of inclination of the avatar during the lifting gesture. A forward inclination (positive offset) is applied during the first 20 frames, while a backward inclination (negative offset) is applied in the remaining ones.

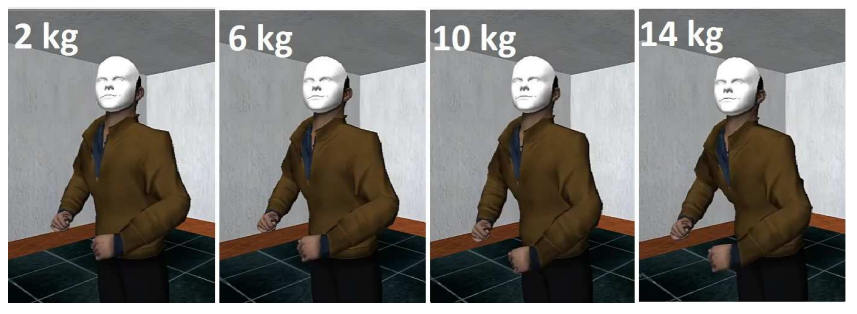

Fig. 5. Forward inclination of the avatar for the four different weights considered in the experiment.

Control refers to the lifting motion of the user while Display refers to the lifting animation of the avatar. In this way, a heavy weight will be perceived as a slower lifting animation of the avatar. Therefore, the user will amplify their own motion in order to lift the virtual object. When the user is lifting a virtual dumbbell, the amplification of his motion is controlled using the following equation:

$$
P=P_{0}+\left(P_{F}-P_{0}\right) \frac{Y-Y_{0}}{k \cdot Y_{F}-Y_{0}}
$$

where $P$ is the posture of the avatar that is associated to the vertical wrists position of the user $Y$ (obtained with the Kinect sensor). $Y_{0}$ and $Y_{F}$ are the initial and maximum vertical wrists position (y-coordinate) of the user motion. $P_{0}$ and $P_{F}$ are the initial avatar posture and final avatar postures of the lifting motion for a specific weight. The factor $k$ is used to control the amplification of the user motion. A larger factor would produce a slower avatar animation and a larger user motion.

\section{USER STUDY}

A user evaluation was conducted to determine the potential of the proposed visual effects and their influence on weight perception. In the evaluation, participants were asked to discriminate the weight of virtual dumbbells by performing a lifting gesture while observing their virtual avatar performing the same gesture. We considered the three visual effects proposed and also, as they can be combined, all the possible combinations (seven in total).

\subsection{Participants}

Eleven volunteers took part in the study, aged from 23 to 30 $($ mean=26.9, $\mathrm{sd}=2.5), 6$ males and 5 females.

\subsection{Apparatus}

The virtual scenario containing the avatar (see Figure 6) was displayed in a 50" inch screen with a resolution of 1920 x 1080 pixels. This 


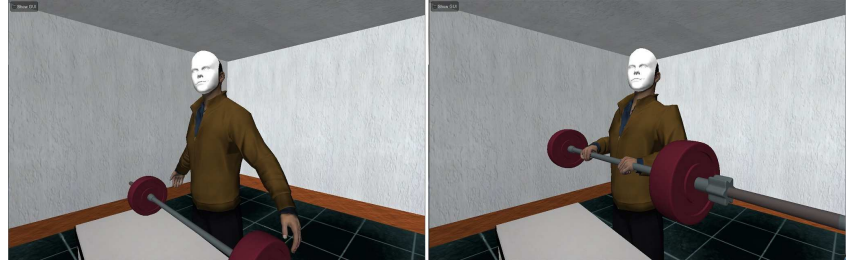

Fig. 6. Virtual scene and avatar used in the weight discrimination task. Left, initial configuration and posture of the avatar. Right, user's gestures are being captured and transferred to the self-animated avatar in real-time.

virtual scenario was implemented using Unity $3 \mathrm{D}^{1}$ and the frame rate was stable at 60 FPS. The same avatar was used for every participant. A white mask was worn by the avatar to provide a neutral cue in the face. In the real scenario, a wood stick on a small table was used to simulate the virtual dumbbell. Thus, participants can adapt more easily to the task. Microsoft Kinect ${ }^{2}$ was used to capture and render the participants gestures in the animated avatar in real-time.

\subsection{Procedure}

Users were instructed to perform a weight discrimination task. In this task, users had to lift several virtual dumbbells performing real lifting gestures. At the same time, users must observe the animation of the avatar inside the virtual environment. We used a wood stick (a real prop representing the virtual dumbbell) in order to allow for a more realistic lifting gesture. The virtual environment consisted of a virtual room in which a self-animated avatar was standing in front a virtual table with a virtual dumbbell on it (see Figure 6). Users were in front a large screen which allowed them to see the virtual environment and observe the animation of the avatar which was driven by their gestures (see Figure 7). During all the test users' gestures were monitored with a Kinect and transferred in real-time to the self-animated avatar. For each lifting operation, users had to lift the virtual dumbbell until a visual indicator (change of color of the face of the avatar) was displayed.

For each discrimination task, users had to sort a group of four virtual dumbbells according to their perceived weight. The sorting task consisted of three iterations: in the first iteration, the user had to lift sequentially each dumbbell observing the animation played by his avatar. After the user had lifted all four dumbbells, the user had to say the number of the heaviest virtual dumbbell. The selected virtual dumbbell was then removed from the group and only three dumbbells remained for the second iteration. The user had to repeat this process two times more in order to sort all the virtual dumbbells.

Finally, at the end of the weight discrimination task, a short questionnaire was given to each participant in order to evaluate their subjective perception and preferences regarding the proposed visual feedbacks. Each participant performed the experiment in one session lasting around 40 minutes.

\subsection{Design and Hypotheses}

The experiment had a within-subject design with one the independent variable: the visual effect. We considered the seven potential combinations between the three visual effects proposed: (CD) the Control/Display ratio of the avatar motion, (MP) the motion profile of the avatar animation and (AI) the angle of inclination of the avatar, and all the possible combinations $(\mathrm{AI}+\mathrm{MP}),(\mathrm{CD}+\mathrm{AI}),(\mathrm{CD}+\mathrm{MP})$ and $(\mathrm{CD}$ $+\mathrm{AI}+\mathrm{MP})$. In order to minimize ordering effects, we employed a Latin square design. Participants did seven repetitions for each visual feedback resulting in 49 trials. For each trial, users were not informed about the visual feedback employed. Considering that each ordering task required 7 lifting gestures, each participant performed 441 lifting gestures.

\footnotetext{
${ }^{1}$ http: //www. unity3d.com

${ }^{2}$ http://www.microsoft.com/en-us/kinectforwindows/
}
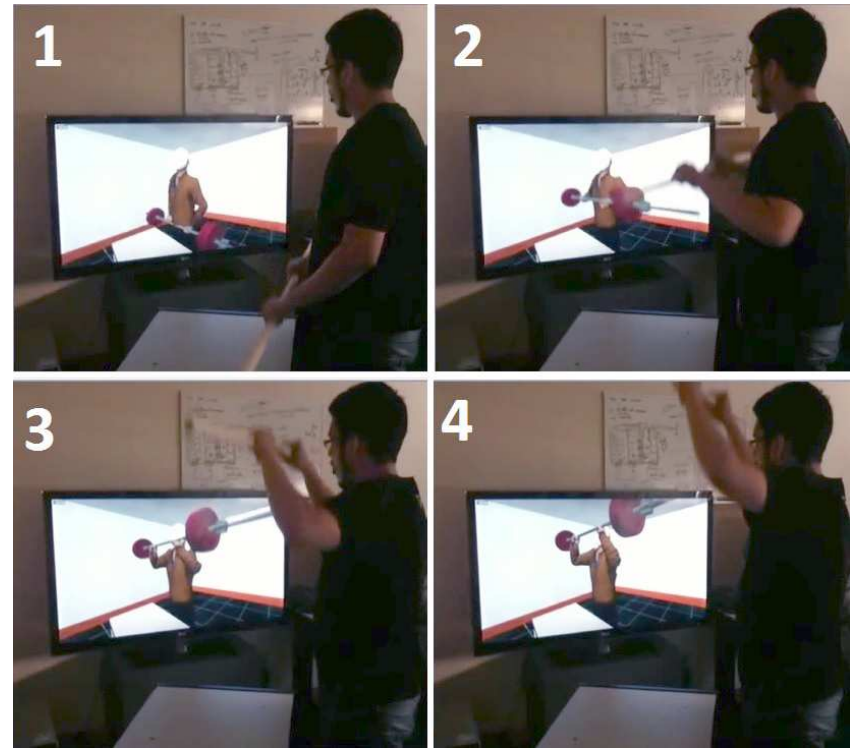

Fig. 7. User's lifting gestures. The animation of the avatar corresponds to the user's lifting gesture and to the weight of the virtual dumbbell. First image, a user is starting to lift the virtual dumbbell. Second and third images, a user is lifting the virtual dumbbell while observing the animation of the avatar. Fourth image, a user has reached the maximum lifting height.

Regarding the different weights used for each task, four virtual weights were simulated. The simulated weights corresponded with the weights of the motion capture data used for the MP condition $2 \mathrm{~kg}, 6 \mathrm{~kg}$, $10 \mathrm{~kg}$ and $14 \mathrm{~kg}$. For the other conditions (AI and $\mathrm{CD})$ the computation of the simulation parameters was computed accordingly. While for the MP condition, each weight corresponded to a different motion profile, for the AI condition, the offset value of the initial inclination is $i_{0}=0$ for all weights. The offset value of the final forward/backward inclination $i_{F}$ for each weight were: $2 \mathrm{~kg}= \pm 0.25,6 \mathrm{~kg}= \pm 0.5,10 \mathrm{~kg}= \pm 0.75$ and $14 \mathrm{~kg}= \pm 1.0$. For the Control/Display ratio approach, the CD ratios used were $2 \mathrm{~kg}=1,6 \mathrm{~kg}=1.06,10 \mathrm{~kg}=1.12$ and $14 \mathrm{~kg}=1.18$. When different visual effects were combined the parameters were chosen consistently (e.g. a motion profile of $2 \mathrm{~kg}$ with a CD ratio of $2 \mathrm{~kg}$ ).

Regarding the data recorded during the experiment, the dependent variables were the ordering error and ordering time. Both were computed for each discrimination task. In order to compute the ordering error for each task, we compared the correct ordering with the ordering determined by the user. The ordering error (E) was computed using Equation 5,

$$
E=\sum_{i=1}^{4}\left|D_{i}-D_{i}^{*}\right|^{2}
$$

where $D_{i}$ is the position of the $\mathrm{i}$-th virtual dumbbell in the correct ordering, and $D_{i}^{*}$ is the position of the $\mathrm{i}$-th virtual dumbeell in the user's ordering. For comparisons purposes, a totally incorrect sorting (inverted order) will obtain an ordering error of 20 , while a totally correct sorting will obtain an error of 0 . The ordering time considered the time spent during the three iterations for each discrimination task. Users were informed that it was preferred a lower ordering error rather than a smaller ordering time.

According to our design, the hypotheses for the experiment were:

- H1: The discrimination accuracy will increase while combining several visual effects.

- H2: The discrimination accuracy will be the same for the three proposed visual effects. 

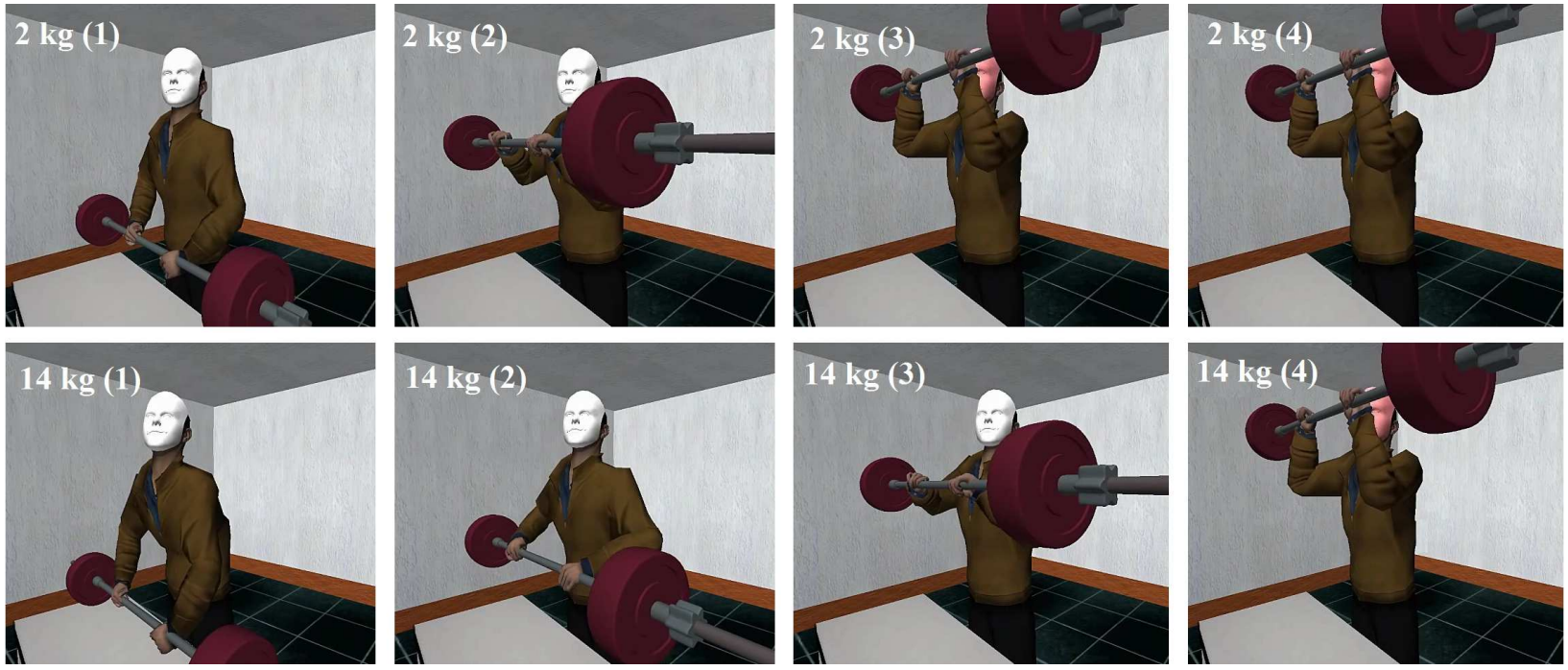

Fig. 8. Sample postures showing all visual effects combined (Control/Display ratio + angle of inclination + motion profile) for the virtual dumbbells of $2 \mathrm{~kg}$ (top) and $14 \mathrm{~kg}$ (bottom). For both sequences the user performed the same (monotonic) lifting gesture. The color change of the face of the avatar showed the maximum height of the virtual lifting motion. As the control display ratio increases the user must perform a wider lifting gesture.

- H3: Users will require the same time to order the four dumbbells for each visual effects.

\subsection{Results}

\subsubsection{Ordering error}

The data (ordering error) for each user were analyzed through a oneway repeated measures ANOVA. Post-hoc comparisons were performed using Bonferroni method with an $(\alpha=0.05)$.

Results showed a main effect of Visual Feedback factor $(F(6,60)=$ 64.07; $p<0.001)$. Post-hoc tests showed that there were significant differences between the different levels of Visual Feedback (see Figure 9). Pairwise tests showed that CD was the Visual Feedback which resulted in significantly lower mean ordering errors, being the combinations $(\mathrm{CD}+\mathrm{MP}+\mathrm{AI})$ and $(\mathrm{CD}+\mathrm{MP})$ the ones obtaining the lowest ordering error scores. However, there was no significant difference between all conditions using the $\mathrm{CD}$ ratio visual feedback $(\mathrm{CD}$, $\mathrm{CD}+\mathrm{MP}, \mathrm{CD}+\mathrm{MP}+\mathrm{AI}, \mathrm{CD}+\mathrm{AI})$. On the contrary, the condition showing the worst ordering score was MP, followed by (AI+MP) and (A), differences among them were significant.

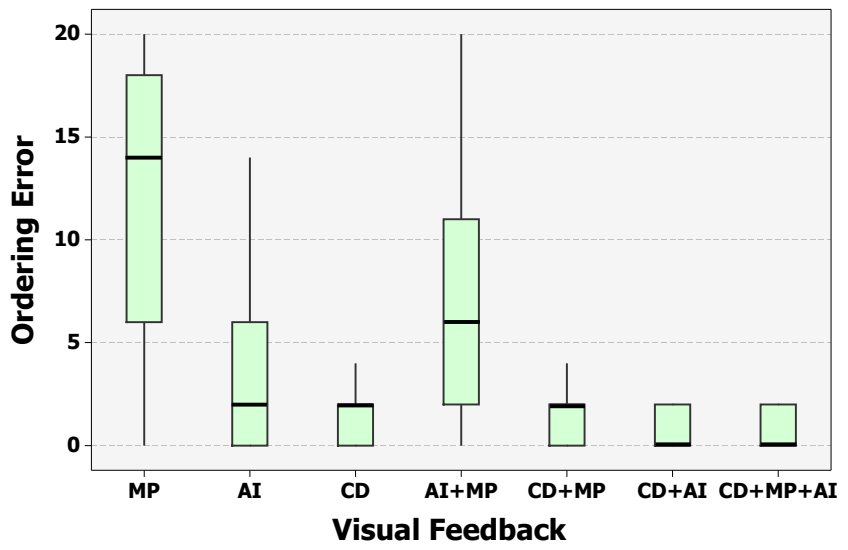

Fig. 9. Box plots for ordering error of the proposed visual conditions. Lower dispersion of ordering errors were obtained by the combination of all visual feedbacks $(C D+M P+A l)$.

\subsubsection{Ordering time}

The mean ordering time for all discrimination tasks was $29.56 \mathrm{sec}-$ onds with a standard deviation of 6.57 seconds. Ordering time for each condition was also analyzed using a one-way ANOVA. The ANOVA showed a main effect of Visual Feedback on ordering time $(F(6,60)=2.30 ; p<0.05)$. Post-hoc tests only showed a significant difference between the $\mathrm{AI}$ and the $\mathrm{CD}+\mathrm{MP}+\mathrm{AI}$ conditions $(p<0.05)$, with a faster ordering time for the AI condition, thus rejecting $\mathbf{H 3}$. However, although there was a significant difference, the difference of mean ordering time is less than 2 seconds (from $30.4 \mathrm{~s}$ to $28.5 \mathrm{~s}$ ).

\subsubsection{Questionnaires}

After the end of the experiment, a questionnaire was given to users in order to know their preferences. First, we asked them whether it was difficult to perceive the differences between weights. $82 \%$ of the users reported no difficulties to find the heaviest weight while $18 \%$ of the users reported difficulties.

Users were also asked to sort the seven visual effects according to the difficulty of the weight perception task. Most of the users $(55 \%)$ preferred the combination of all visual effects $(\mathrm{CD}+\mathrm{AI}+\mathrm{MP}), 18 \%$ of the users preferred the combination of the $C D$ ratio with the angle of inclination, $18 \%$ of the users preferred the $C D$ ratio, and $9 \%$ of the users preferred the angle of inclination. Users were also asked to sort the main visual effects used (CD, AI or MP) according how helpful they were to determine the differences between weights. $55 \%$ of the users preferred the $C D$ ratio, while $45 \%$ of the users preferred the angle of inclination.

Furthermore, we were interested in knowing if the users found the avatar animation helpful. Most of the users (91\%) found helpful the animation, while $9 \%$ of the user did not find the animation helpful. Finally, $82 \%$ of the users reported that they felt tired at the end of the experiment while $18 \%$ of the users did not reported any fatigue.

\subsection{Discussion}

The main purpose of the experiment was to test the perception of weight lifting using our three different visual effects and their combination. Results suggest that people are able to rank the different weights of dumbbells by exclusively relying on visual effects of the avatar animation. This means that our approach is valid and can succeed in simulating haptic percepts by exclusively relying on visual effects related to avatar's motions or posture. However, we observed performance differences among the different techniques evaluated, thus rejecting [H1]. 
Results showed that the effect of the $C D$ ratio was the strongest visual cue since the ordering errors were minimal, especially compared to the other visual effects (angle of inclination and motion profile) (see Figure 9). The mean ordering error was 2.442 units which represents that users made (in average) a missclassification of order one per trial. This means that different weight properties in a avatar-based interactive virtual environment can be effectively simulated by modifying the Control/Display ratio of the avatar motion. These results are consistent with previous works $[5,15]$ which also used the $C / D$ ratio in order to create a visuo-haptic illusion. Altering the C/D ratio between users' gestures and the animation of the avatar is a solid cue and thus could be further used.

Second, although the performance for angle of inclination is less efficient, it still enables the user to perceive the differences in the virtual dumbbell. The mean ordering error for AI was 3.571 which means that participants in overall made two missclassifications of order one or one of order two for each trial. This results shows that while participants were more efficient to distinguish bigger differences in the angle of inclination than smaller ones. Interestingly, according to the questionnaires, half of the users relied mostly on this cue when available. This suggests that although the angle of inclination was less efficient than the CD ratio, participants took into account the avatar posture for the discrimination task. We can conclude that the only altering the animation of the spine was not enough to deliver a strong visual cue. Thus a more complex approach should be considered in order to provide better animations in order to deliver a better pseudo-haptic effect.

Last, our results suggest that the motion profile cue is working less efficiently. The mean ordering error was 11.455 units which corresponds to error orders of two or three for each trial. This bad performance of the motion profile can be explained by the fact that participants could have interpreted the slow response of the motion profiles of $10 \mathrm{~kg}$ and $14 \mathrm{~kg}$ by a latency of the tracking system. According to the results, participants were only able to discriminate the weight of the virtual dumbbells when using it in combination with the $C D$ ratio and the angle of inclination. Our participants found it actually easier to perceive the motion profile information with a corresponding $C D$ ratio and a consistent angle of inclination. Furthermore, most users found that the changes between the different motion profiles were too small or too subtle. As future work we could thus test stronger values and stronger differences in motion profiles so to generate more perceptible sensations. Differences in performance between techniques makes us reject [H2].

Nevertheless, the combination of all proposed visual effects obtained the lowest ordering error compared with each separated visual feedback. This suggests that our visual effects can be combined and integrated without producing conflicts. This results is also supported by the fact that the combination of the three visual effects was the preferred visual cue among the users.

Finally, regarding fatigue, most of the participants (82\%) reported that they were tired after performing the 441 lifting motions (mass of the tangible object was less than $200 \mathrm{~g}$ ) in 40 minutes. Fatigue is a complex phenomenon which involves peripherals (within muscles) to central (cognitive) fatigues. There was no speed constraint and the subject could spend enough time between trials to recover from peripheral fatigue with such low mass. Addressing fatigue would be really interesting in future works, especially to evaluate if fatigue increases when force is more accurately perceived, as suggested by [2]. It would require standardized protocols to evaluate fatigue at various levels of the pyramidal control system. In the present study, the questionnaire provided us with a global feedback which cannot enable us to conclude about this assumption.

\section{CONCLUSION AND FUtURE WORK}

In this paper, we have proposed and evaluated pseudo-haptic avatar interaction: physicalizing avatar-based interaction. In the proposed approach, the mapping between user's gestures and the avatar, and the avatar animation on itself are used to simulate physical interaction with the virtual environment. The proposed approach was evaluated in a weight discrimination task. In this task, the user lifted a virtual dumb- bell by mapping gestures in real-time onto a self-animated avatar. The avatar shows different visual effort related to the weight of each virtual dumbbell. Three different pseudo-haptic effects were evaluated: the motion profile obtained from motion capture data, adapting the avatar posture (the angle of inclination of the avatar), and modulating the $\mathrm{C} / \mathrm{D}$ ratio of the avatar motion. The results showed that the C/D ratio approach was the one obtaining the better performance followed by the angle of inclination and the motion profile. Furthermore, in order to obtain and improved feedback, the three approaches can be combined, which resulted in the best weight discrimination accuracy.

As future work, we would like to extend this work to other types of interactions that require various types of forces. In ergonomics it would help users to better adapt their motion to various workstation configurations with various force strategies. Thus it could help to adapt future workstations to decrease the forces required to perform the imposed tasks. Without force feedbacks, users and ergonoms may fail to converge to an efficient workstation. Designing specific haptic devices for a large set of operator's actions is almost impossible and would required numerous specific systems. Using pseudo-haptic paradigms would help to tackle this problem. Another typical application is to train users to complex motor tasks such as maintaining complex, dangerous or costly systems. Again, in most of the cases, experiencing force-based interactions in virtual environment for such various movements and tools, would require developing numerous specific haptic devices.

In sports, many motions involve physical interactions with the environment (such as performing push-ups), objects (such as hitting a ball with a tennis racket) and opponents (such as contacts in rugby). Most of these physical interactions are difficult to simulate in VR, because it would require complex and specific devices, especially capable of simulating high-frequency and high-intensity forces. Using pseudohaptic paradigms would help to address a wide range of interactions in sports without using complex or inappropriate devices. With the development of exergames dedicated to the wide public, users wish to experience sports at home thanks to immersive systems. The key idea is to make people practice physical exercises in high-motivational interactive games. However, current games are limited to physical activities with limited physical interaction with the world because it would require specific devices, such as Nintendo Balance board, or using real sports devices (such as dumbbells, elastics...). Using pseudo-haptic paradigms could help to address new types of physical activities without requiring complex and specific devices.

\section{REFERENCES}

[1] The Effect of Viewing a Self-Avatar on Distance Judgments in an HMDBased Virtual Environment. Presence: Teleoperators and Virtual Environments, 19(3):230-242, 2010.

[2] Y. Ban, T. Narumi, T. Fujii, S. Sakurai, J. Imura, T. Tanikawa, and M. Hirose. Augmented endurance: controlling fatigue while handling objects by affecting weight perception using augmented reality. In CHI 2013, pages 69-78, 2013.

[3] S. Benford, J. Bowers, L. E. Fahlén, C. Greenhalgh, and D. Snowdon. User embodiment in collaborative virtual environments. In Proceedings of the SIGCHI conference on Human factors in computing systems, pages 242-249, 1995.

[4] T. Dodds, B. Mohler, and H. Bülthoff. A Communication Task in HMD Virtual Environments: Speaker and Listener Movement Improves Communication. In 23rd Annual Conference on Computer Animation and Social Agents (CASA 2010), pages 1 - 4, 2010.

[5] L. Dominjon, A. Lécuyer, J. Burkhardt, P. Richard, and S. Richir. Influence of control/display ratio on the perception of mass of manipulated objects in virtual environments. In IEEE International Conference on Virtual Reality, pages 19-25, 2005.

[6] R. M. Ehrig, W. R. Taylor, G. N. Duda, and M. O. Heller. A survey of formal methods for determining the centre of rotation of ball joints. Journal of biomechanics, 39(15):2798-2809, 2006.

[7] M. O. Ernst and M. S. Banks. Humans integrate visual and haptic information in a statistically optimal fashion. Nature, 415(6870):429-433, 2002. 
[8] T. Hachisu, G. Cirio, M. Marchal, A. Lécuyer, and H. Kajimoto. Pseudohaptic feedback augmented with visual and tactile vibrations. In Proceedings of International Symposium on VR Innovations, pages 331-332, 2011.

[9] J. G. Hamilton. Identifying with an avatar: a multidisciplinary perspective. In In Proceedings of the Cumulus Conference: 380 South: Hemispheric Shifts Across Learning, Teaching and Research, 2009.

[10] M. A. Heller, J. A. Calcaterra, S. L. Green, and L. Brown. Intersensory conflict between vision and touch: The response modality dominates when precise, attention-riveting judgements are required. Perception Psychophys, 61:1384-1398, 1999.

[11] L. Hoyet, F. Multon, A. Lécuyer, and T. Komura. Can we distinguish biological motions of virtual humans? perceptual study with captured motions of weight lifting. In ACM Symposium on Virtual Reality Software and Technology, 2010.

[12] M. Karam and M. C. Schraefel. A taxonomy of gestures in human computer interaction. In Technical report, University of Southampton, 2005.

[13] K. Kilteni, I. Bergstrom, and M. Slater. Drumming in immersive virtual reality: the body shapes the way we play. IEEE Transactions on Visualization and Computer Graphics, 19(4):597-605, 2013.

[14] R. L. Klatzky, S. Lederman, and C. Reed. There's more to touch than meeets the eye: The salience of object attributes for haptics with and without vision. Journal on Experimental Psychology: General, 116:356369, 1987.

[15] A. Lécuyer. Simulating haptic feedback using vision: A survey of research and applications of pseudo-haptic feedback. Presence: Teleoperators and Virtual Environments, 18:39-53, 2009.

[16] A. Lécuyer, J. M. Burkhardt, S. Coquillart, and P. Coiffet. Boundary of illusion: An experiment of sensory integration with a pseudo-haptic system. In Proceedings of the IEEE International Conference on Virtual Reality, pages 115-122, 2009.

[17] A. Lécuyer, S. Coquillard, A. Kheddar, P. Richard, and P. Coiffet Pseudo-haptic feedback: Can isometric input devices simulate force feedback? In Proceedings of the IEEE International Conference on Virtual Reality, pages 83-90, 2000.

[18] B. Lenggenhager, T. Tadi, T. Metzinger, and O. Blanke. Video ergo sum: manipulating bodily self-consciousness. Science, 317(5841):10969, 2007.

[19] S. Linkenauger, M. Leyrer, H. H. Blthoff, and B. J. Mohler. Welcome to wonderland: The influence of the size and shape of a virtual hand on the perceived size and shape of virtual objects. PLOS ONE, 8(7):1-16, 2013.

[20] D. Maupu, R. Boulic, and D. Thalmann. Characterizing full-body reach duration across task and viewpoint modalities. JVRB - Journal of Virtual Reality and Broadcasting, 5(2008)(15), 2008.

[21] T. Ogawa and Y. Kambayashi. Physical instructional support system using virtual avatars. In Proceedings of the 2012 International Conference on Advances in Computer-Human Interactions, pages 262-265, 2012.

[22] S. Okamoto, H. Kawasaki, H. Iizuka, T. Yokosaka, T. Yonemura, Y. Hashimoto, H. Ando, and T. Maeda. Inducing human motion by visual manipulation. In Proceedings of the 2nd Augmented Human International Conference, page 32, 2011.

[23] H. Omosako, A. Kimura, F. Shibata, and H. Tamura. Shape-COG illusion: Psychophysical influence on center-of-gravity perception by mixedreality visual stimulation. In IEEE Virtual Reality Short Papers and Posters (VRW), pages 65-66, 2012.

[24] A. Pusch, O. Martin, and S. Coquillart. HEMPhand-displacement-based pseudo-haptics: A study of a force field application and a behavioural analysis. International Journal of Human-Computer Studies, 67(3):256268, 2009.

[25] I. Rock and J. Victor. Vision and touch: An experimentally created conflict between the two senses. Science, 143:594-596, 1964.

[26] M. Slater, D. Perez-Marcos, H. Ehrsson, and M. V. Sanchez-Vives. Towards a digital body: the virtual arm illusion. Frontiers in Human Neuroscience, 2(6), 2008.

[27] M. Slater, D. Perez-Marcos, H. H. Ehrsson, and M. V. Sanchez-Vives Inducing illusory ownership of a virtual body. Frontiers in neuroscience, 3(2):214-20, Sept. 2009.

[28] M. A. Srinivasan, G. L. Beauregard, and D. L. Brock. The impact of visual information on the haptic perception of stiffness in virtual environments. In Proceedings of the 5th International Symposium on Haptic Interfaces for Virtual Environment and Teleoperator Systems, pages 555559, 1996.

[29] G. Wu, F. C. Van der Helm, H. Veeger, M. Makhsous, P. Van Roy, C. An- glin, J. Nagels, A. R. Karduna, K. McQuade, X. Wang, et al. Isb recommendation on definitions of joint coordinate systems of various joints for the reporting of human joint motionpart ii: shoulder, elbow, wrist and hand. Journal of biomechanics, 38(5):981-992, 2005. 